\title{
Immunomagnetic enrichment of circulating tumor cells prior to tumor Ig specific qASO-PCR enhances the sensivity of minimal residual disease detection in multiple myeloma.
}

\author{
Wouter De Brouwer, Jona Vanderstraeten, Christian Demanet, Rik Schots, Marleen Bakkus* and Ivan Van \\ Riet $^{*}$ \\ UZ Brussel - Vrije Universiteit Brussel. * = equal contributions
}

\section{Relevance:}

-Multiple myeloma is 2nd most common hematological malignancy

- New therapies give better responses and prolonged survival but still relapse after complete remission (CR)

-Presence of minimal residual disease (MRD) in bone marrow of MM patients is associated with worse prognosis

-Tumor monitoring using bone marrow (BM) biopsies is uncomfortable for the patient with risk of false negative results and risk of missing tumor heterogeneity.

\section{Questions:}

- Is detection of circulating tumor cells CTC's in MRD setting possible and how can the detection sensitivity be increased?

- Might the detection of CTC's in treated MM patients be an alternative for repeated BM biopsies?

-Is the detection of CTC's in treated MM patients associated with worse prognosis?

\section{Aim of the study:}

To develop and validate a dual platform method to measure MRD in MM, based on immunomagnetic enrichment of (CD138+) CTC's followed by molecular tumor detection using allele-specific oligonucleotide polymerase chain reaction ( qASO$\mathrm{PCR})$.

\section{Methods:}

A series of spike-in experiments were performed using a fixed amount of peripheral blood mononuclear cells (PBMC's) contaminated with LP-1 cells (human a myeloma cell line derived from peripheral blood ) in decreasing concentrations (10-2 to 10-6) (figure 1a).

CTC's (LP-1 cells) were enriched using the AUTO-Macs device with CD138microbeads (Miltenyi)

DNA was extracted from enriched CTC (LP-1 cells) fractions and paired non-enriched (PBMC'S + LP-1 cells) cell suspensions

Paired samples were analysed by allele-specific oligonucleotide polymerase chain reaction (qASO-PCR) to determine the contamination level with LP-1 cells

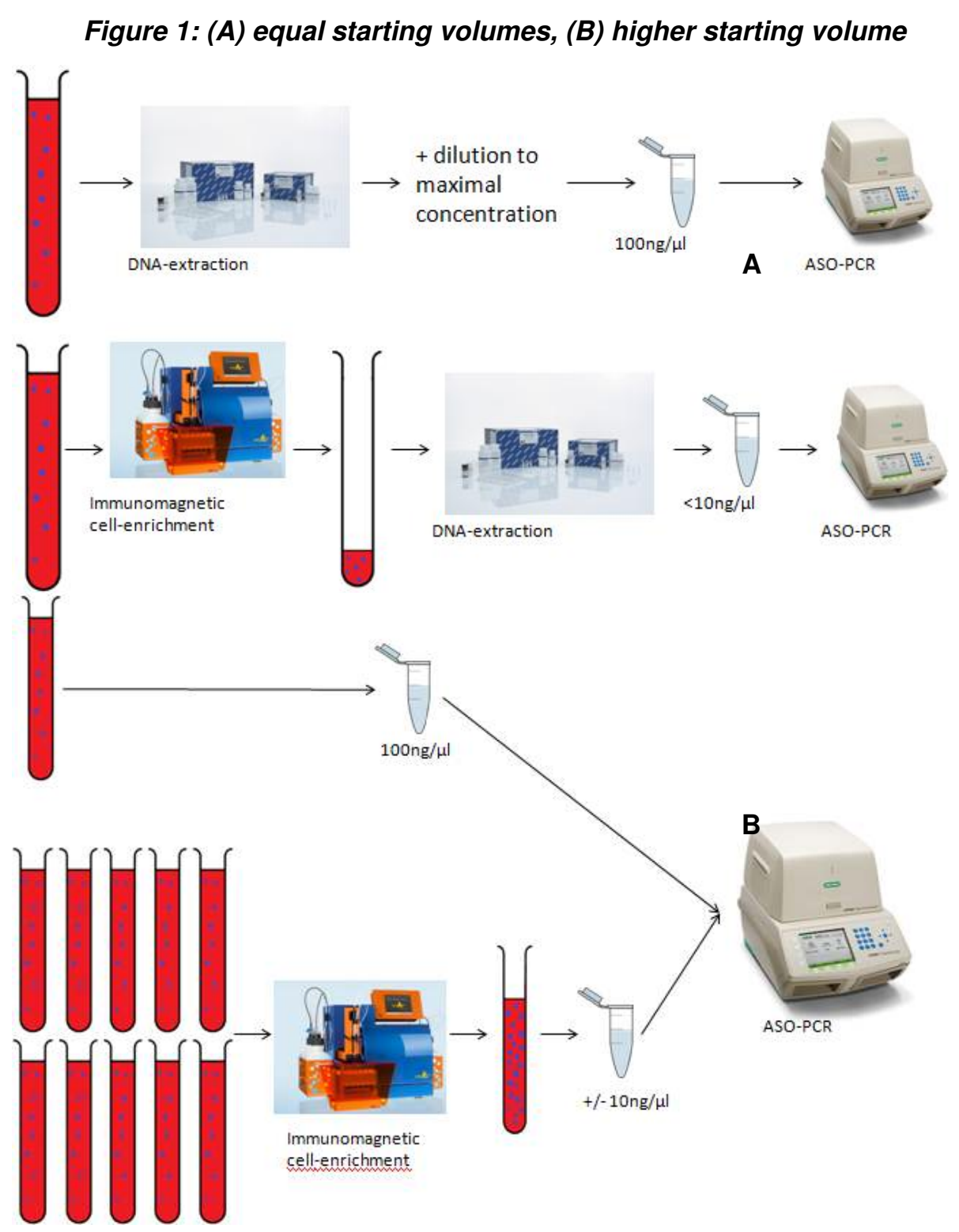

Results:

-Immunomagnetic isolation of CD138+ circulating tumor cells results in tumor cell enrichment up to 150-fold (figure 2) but offers no additional value in tumor detection level when a standard blood sample volume of $10 \mathrm{ml}$ is used. Using the qASO-PCR method both enriched CTC fractions as well as unseparated PBMC suspensions reveal a tumor detection limit of 10-5

-The sensivity of qASO-PCR is limited by a maximal DNA input of $100 \mathrm{ng} / \mathrm{\mu l}$ (corresponding with approximately 165.000 cells)

-As the immunomagnetic enrichment procedures allows for higher starting volumes, a 10 times higher starting volume $(100 \mathrm{ml})$ can result in cell numbers far below the technical limits of qASO-PCR (figure $1 \mathrm{~b}$ )

- Using higher starting volumes for the CTC enrichment procedure can result for comparable tumor contamination in an increase of tumor detection sensivity up to 10fold $\left(10^{-6}\right)$ (table 1)

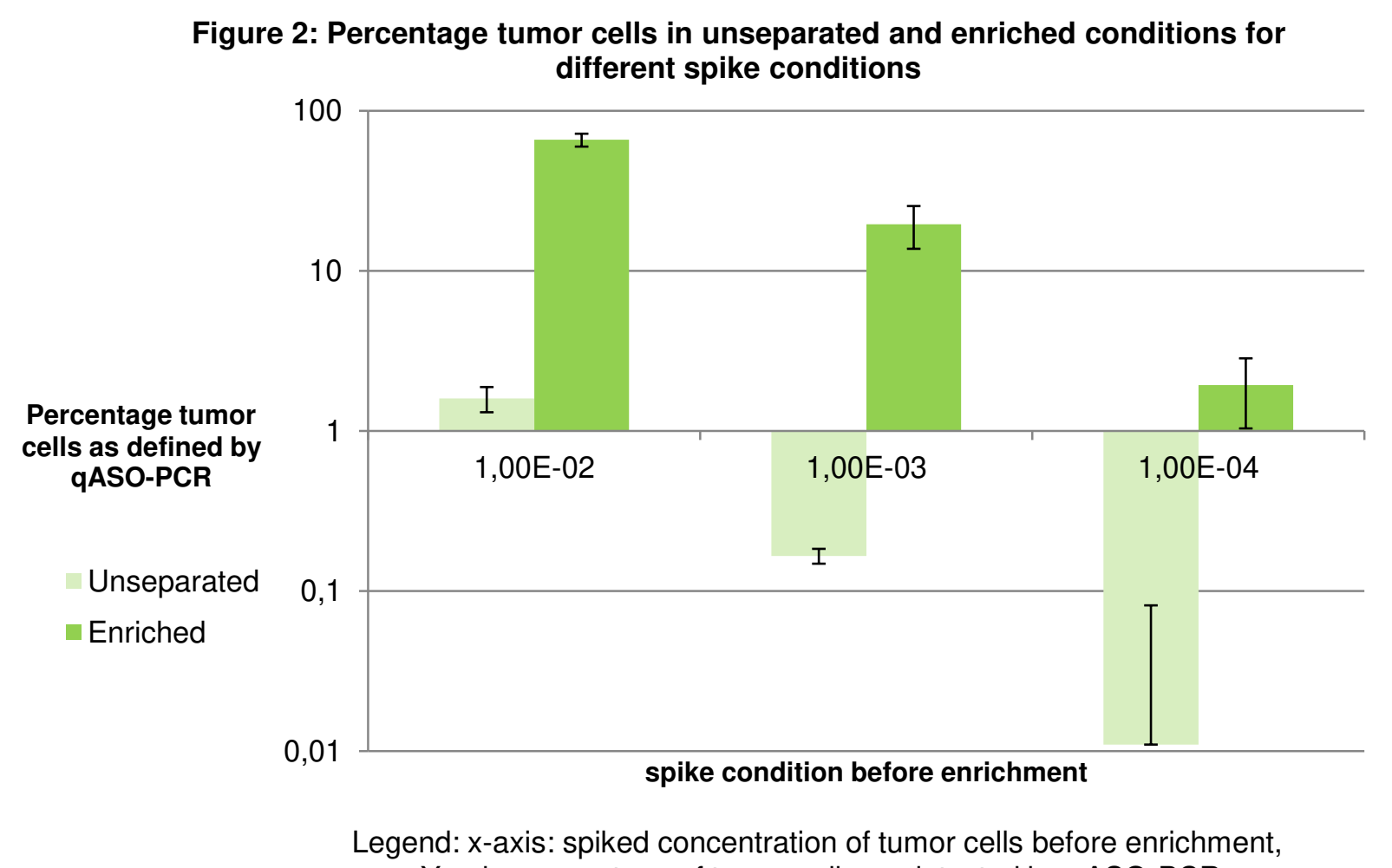
$Y$-axis: percentage of tumor cells as detected by qASO-PCR

Table 1: CTC detected by ASO-PCR

\begin{tabular}{|lll|}
\hline Concentration & Unseparated $(100 \mathrm{ng} / \mu \mathrm{ll})$ & Enriched $(10 \mathrm{ng} / \mu \mathrm{ll})$ \\
\hline $10^{-5}$ & $9 / 9$ & $9 / 9$ \\
\hline $10^{-6}$ & $3 / 9(p<0.05)$ & $9 / 9$ \\
\hline $10^{-7}$ & $2 / 9$ (NS) & $3 / 9$ \\
\hline
\end{tabular}

Legend: This table shows the detection rate for not-enriched (unseparated) and enriched fractions respectively. Concentrations between brackets:DNA concentration (max 100ng/Ml).

\section{Conclusions:}

Our data indicate that CTC enrichment prior to molecular tumor detection allows to analyze larger blood sample volumes and thus increasing the sensitivity of qASO-PCR analysis by a factor ten to $10^{-6}$. Compared with the standard qASO-PCR technique with non-enriched cell suspensions, our method allows for more tumor cells to be analyzed. Future experiments have to reveal whether the enrichment efficiency of CTC (in terms of purity and yield) can be increased and whether the use of Igtargeted NGS can further improve the tumor detection sensitivity in this already promising dual-platform strategy 